\title{
Internal Ballistics Model and Analysis of Rod-less Cylinder Ejection Device Based on real gas effect
}

\author{
Lin $\mathrm{Yao}^{1,}$, , Dawei Ma ${ }^{1, b}$ and Guigao Le $e^{1, c}$ \\ ${ }^{1}$ School of Mechanical Engineering, Nanjing University of Science and Technology, Nanjing \\ 210094, China \\ ayaolinxueshu@163.com, ${ }^{\mathrm{b}}$ ma-dawei@mail.njust.edu.cn, ${ }^{\mathrm{c}}$ le-guigao@mail.njust.edu.cn
}

Keywords: rod-less cylinder; real gas effect; dynamics analysis; internal ballistics model; ejection device.

Abstract. In the launching processes of Rod-less Cylinder ejection device, the pressure is much higher than the air critical pressure, and the temperature can deviate significantly from the Boyle temperature. In such situations, the thermo-physical properties and pneumatic performance can't be described accurately by the ideal gas law. The mathematical expression of pneumatic interior ballistics model are deduced on the basis of the 'RK' state equation for the lifting cylinder. The interior ballistics equations are solved by using MATLAB software. The change rule of thermodynamic and kinematics parameters are obtained in the process of catapult. The comparison results show that the real gas effect accelerates the pressure decreasing rates in the hyperbaric pressure chamber and decelerates the increasing rates in the low pressure chamber. Besides, the real gas effect reduces the missile launching speed under the same piston stroke. The results of numerical calculation can provided reference for prototype design.

\section{Introduction}

At present, the compressed air cold ejection technology application is more and more widely. Compared to gun power launch technology, high pressure air has the advantages of no pollution, no thermal protection, high power density, cheapness, reliable performance, recycling use, and being easy to maintain internal ballistic[1]. It also has been applied to industrial automation, robot driving, compressed air powered vehicles, and even some special industries such as aeronautics, astronautics, and weapons design[2].

Traditionally, the study of ejection performance and internal ballistic analysis are based on the ideal gas state equations[3]. However, in high-pressure pneumatic dynamic thermodynamic processes, the pressure is much higher than the air critical pressure and the temperature can deviate significantly from the Boyle temperature. Since the thermodynamic properties based on the ideal gas are not authentic, the deviations in the mass and energy balances in thermodynamic process may not be acceptable.

As for the study of launching processes for compressed air cold ejection device, some researchers finish lots of related work. Ren[1] et al. have researched the pneumatic performance of a high pressure ejection device based on real specific energy and specific enthalpy, and the pneumatic performance analysis indicates that the real dynamic thermodynamic process is obviously different from the ideal one. Yang[4] et al. have researched the internal ballistics response characteristics based on the Virial state equation and the result show that the compression factor reaches 1.103. Bai[5] et al. studied a two-step lifting cylinder ejection device. Niu[6] et al. researched the launching process of under-water piston pulling ejection under the coupled calculation.

But above all, the internal ballistics model and analysis of rod-less cylinder cold launching device based on real gas equation were few reports. In the paper, the 'RK' state equation are adopted to simulate the whole ejecting process. 


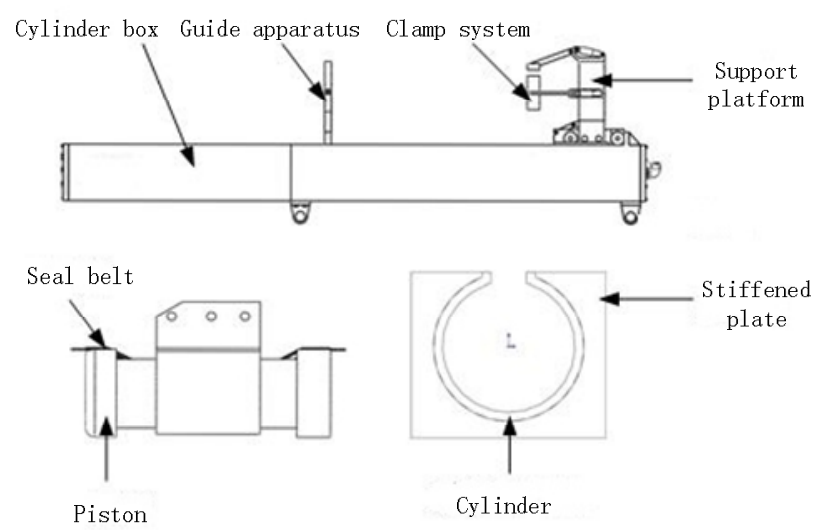

Figure 1. Structure of rod-less cylinder ejection device

\section{Mathematical model}

\section{RK state equations}

As we all know, the real gas state equation-' RK ' that contains only two specific parameter was proposed by Redlich and Kwong in 1949[7]. The equation can be written as:

$$
\begin{aligned}
& p=\frac{R T}{V_{m}-b}-\frac{a}{T^{0.5} V_{m}\left(V_{m}+b\right)} \\
& a=\frac{0.42748 R^{2} T_{c}^{2.5}}{p_{c}}, \quad b=\frac{0.84664 R T_{c}}{p_{c}}
\end{aligned}
$$

In the equation, we will integrate the specific volume from $\mathrm{v}=+\infty$ to $\mathrm{v}$ and then the analytical expressions of specific thermodynamic energy expression and specific enthalpy for real gas are obtained as follows[8]:

$$
u=C_{v i} T+7.401 R_{g} T_{c}^{1.5} T^{-0.5} \ln \left[\frac{V_{m}}{V_{m}+0.08664 R_{g} T_{c} M / p_{c}}\right]
$$

\section{Internal Ballistics Model}

In the course of the pneumatic ejection, taking the subsonic and sonic flow into account, mass flow equation can be written as:

$$
Q_{\mathrm{m}}= \begin{cases}\mu_{x} \frac{p_{1} A}{\sqrt{R_{g} T_{1}}} \sqrt{\frac{2 k}{k-1}\left[\left(\frac{p_{2}}{p_{1}}\right)^{\frac{2}{k}}-\left(\frac{p_{2}}{p_{1}}\right)^{\frac{k+1}{k}}\right]}\left(\frac{2}{k+1}\right)^{\frac{k}{k-1}}<\frac{p_{2}}{p_{1}}<1 \\ \mu_{x} \sqrt{\mathrm{k}}\left(\frac{2}{k+1}\right)^{\frac{k+1}{2(k-1)}} p_{1} A / \sqrt{R_{g} T_{c}} & \frac{p_{2}}{p_{1}} \leq\left(\frac{2}{k+1}\right)^{\frac{k}{k-1}}\end{cases}
$$

Where subscripts 1 and 2 indicate high-pressure chamber and low pressure chamber respectively, $Q_{\mathrm{m}}$ is the mass flow of the high pressure air, $A$ is the cross-sectional area of valve, $\mu_{\mathrm{x}}$ is the flow modification coefficient.

According to mass and energy conservation laws, and the flow equation in the high-pressure chamber and low pressure chamber, the following relations can be established.

Let $X 1=\rho_{1}, X 2=T_{1}, X 3=m_{2}, X 4=T_{2}, X 5=1, X 6=v_{2}$, the closed pneumatic equations can be established as the formula(5).

Where $u$ is the specific thermodynamic energy expression, $h$ is the specific enthalpy, $S$ is the piston area, $m_{e}$ is the mass of missile, $m_{t}$ is the mass of piston, $v_{2}$ is the velocity of piston. 


$$
\begin{aligned}
& \mathrm{X}_{1}=\frac{-\mathrm{Q}_{\mathrm{m}}}{\mathrm{V}_{1}} \\
& \mathbb{X}_{2}=\frac{-\bigotimes_{1}\left[7.401 R_{g} T_{c}^{1.5} X_{2}^{-0.5}\left(\frac{1}{V_{m 1}}-\frac{1}{V_{m 1}+0.08664 R_{g} T_{c} M / p_{c}}\right) \cdot\left(\frac{M}{-X_{1}^{2}}\right)+\frac{\left(u_{1}+h_{1}\right)}{X_{1}}\right]}{\left[C_{v i}-3.7005 R_{g} T_{c}^{1.5} X_{2}^{-1.5} \ln \left(\frac{V_{m 1}}{V_{m 1}+0.08664 R_{g} T_{c} M / p_{c}}\right)\right]} \\
& \mathbb{X}_{3}=Q_{m} \\
& \mathrm{X}_{4}=\frac{\left[-Q_{m} u_{2}-n S p_{2} X_{6}+Q_{m} h_{1}-7.401 R_{g} T_{c}^{1.5} X_{4}^{-0.5}\left(\frac{1}{V_{m 2}}-\frac{1}{V_{m 2}+0.08664 R_{g} T_{c} M / p_{c}}\right) \cdot \frac{M S X_{3} X_{5}-M\left(V_{0}+n S X_{5}\right) Q_{m}}{X_{3}}\right]}{X_{3}\left(C_{v i}-3.7005 R_{g} T_{c}^{1.5} X_{4}^{-1.5} \ln \left(\frac{V_{m 2}}{V_{m 2}+0.08664 R_{g} T_{c} M / p_{c}}\right)\right)} \\
& x_{5}=X 6 \\
& \mathbb{X}_{6}=\frac{\left(p_{2}-p\right) S-1.2\left(m_{e}+m_{t}\right) g}{\left(m_{e}+m_{t}\right)}, \bigotimes_{6}<9.8, \quad \mathbf{X}_{6}=0 \\
& u_{1}=C_{v i} T_{1}+7.401 R_{g} T_{c}^{1.5} T_{1}^{-0.5} \ln \left[\frac{V_{m 1}}{V_{m 1}+0.08664 R_{g} T_{c} M / p_{c}}\right] \\
& u_{2}=C_{v i} T_{2}+7.401 R_{g} T_{c}^{1.5} T_{2}^{-0.5} \ln \left[\frac{V_{m 2}}{V_{m 2}+0.08664 R_{g} T_{c} M / p_{c}}\right] \\
& h_{1}=C_{v i} T_{1}+7.401 R_{g} T_{c}^{1.5} T_{1}^{-0.5} \ln \left[\frac{V_{m 1}}{V_{m 1}+0.08664 R_{g} T_{c} M / p_{c}}\right]+p_{1} V \\
& h_{2}=C_{v i} T_{2}+7.401 R_{g} T_{c}^{1.5} T_{2}^{-0.5} \ln \left[\frac{V_{m 2}}{V_{m 2}+0.08664 R_{g} T_{c} M / p_{c}}\right]+p_{2} V
\end{aligned}
$$

\section{Results and discussion}

Figure 2 shows the laws for temperature in whole launch process. First, from figure 2, we can see that the temperature in the high pressure chamber decreases all the time. Besides, we can know the temperature in the low pressure chamber increases immediately, and then decreases slowly when the missile moves upward. It shows that the real temperature of gas in the low pressure is always lower than ideal one, which indicates that the real gas effects decelerates the temperature increasing rates in the low pressure chamber in the early stage, and accelerates the rate of decrease of the temperature with the missile moving upward.

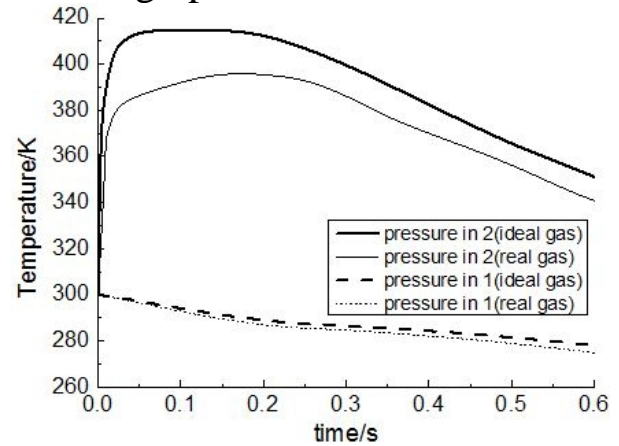

Figure 2. Temperature contrast curve

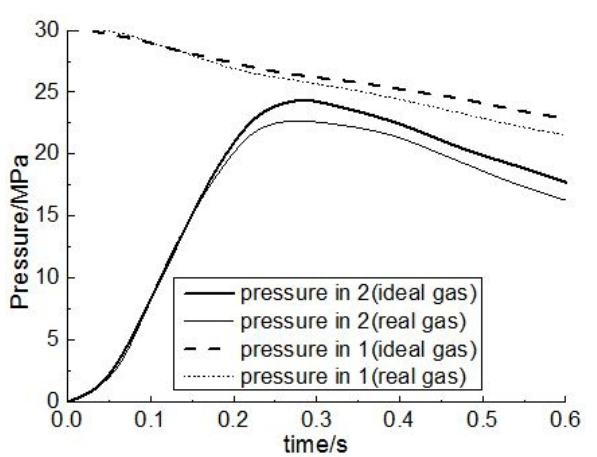

Figure 3. Pressure contrast curve

Figure 3 shows the pressure variations in the high pressure chamber and low pressure chamber. We can see that the temperature in the high pressure chamber continues to decay. The real pressure decay rate in whole launch process is greater than the ideal decay rate. It shows that the real pressure of gas 
in the low pressure is always lower than ideal one, which indicates that the real gas effects decelerates the pressure increasing rates in the low pressure chamber.

Figures 4-5 shows the laws for acceleration and velocity in whole ejection launch process. With the missile overload consistent with the pressure in the low pressure chamber, agreement between the acceleration of missile and pressure in the low pressure chamber are fairly satisfied. From figure 5,we can get the conclusion that the velocity of missile based on an ideal gas is significantly greater than that based on a real gas.

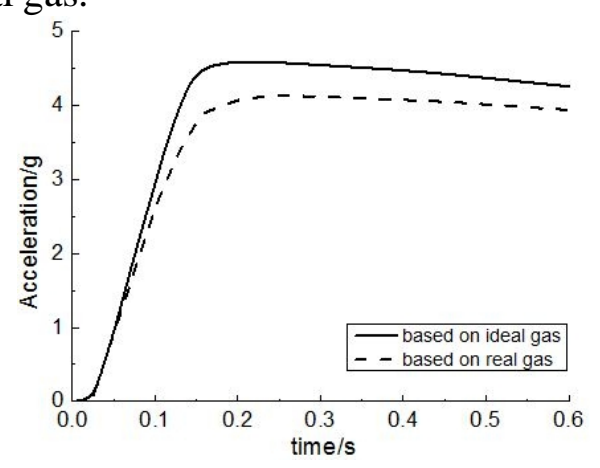

Figure 4. Acceleration contrast curve

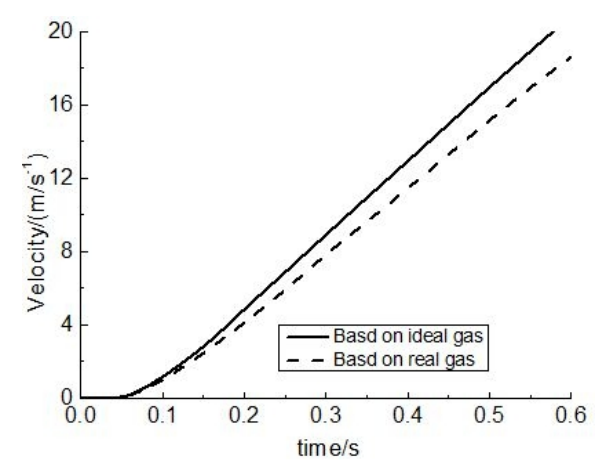

Figure 5. Velocity contrast curve

\section{Conclusions}

In the study, the real gas state equation-'RK' equation is used to describe the interior ballistics properties of high pressure ejection device. The study on dynamic thermodynamic analyses, charging and discharging processes are of particular importance in high pressure air applications. In addition, based on the real gas equation, the internal ballistics mathematical model for the rod-less cylinder pneumatic ejection system is established. Numerical simulations are also performed. The real gas effects accelerate the pressure and temperature rates of decrease in the hyperbaric pressure chamber, and decelerate the rates of increase in the low pressure chamber. So, we can draw the conclusion that the real work capability of hyperbaric air is lower than that of ideal gas. The study reveals the real gas effect during the whole ejection process.

\section{Acknowledgements}

This work was financially supported by the Jiangsu Natural Science Foundation (BK20130761).

\section{References}

[1] REN Jie, MA Da-wei, LE Gui-gao. Pneumatic Performance Study of a High Pressure Ejection Device Based on Real Specific Energy and Specific Enthalpy[J]. Energy, 2014, 16: 4801-4817.

[2] LUO Yu-xi, WANG Xuan-yin. Exergy analysis on throttle reduction efficiency based on real gas equations [J]. Energy, 2010, 35: 181-187.

[3] LUO Yu-xi. A study of the real gas effects on high pressure pneumatics and the basic theoretical and experimental researches of pressure reduction system[D]. Zhejiang University, 2011.

[4] YANG Feng-bo, MA Da-wei, LE Gui-gao. Interior Ballistics Modeling and Calculation of High-pressure Ejection Device [J]. ACTA ARMAMENTARII, 2013, 34(5):527 534.

[5] BAI Peng-ying, QIAO Jun. Analysis about the interior trajectory of Two-step cylinder ejection device[J]. Modern Defence Technology, 2007, 35(4):44 49.

[6] NIU Qing-yong, LI Tian-yun, ZHU Xiang. Sensitivity analysis of the performance parameters of an underwater pneumatic launcher[J]. Journal of Harbin Engineering University, 2015, 36(7):1-5. 
[7] G Soave. Equilibrium Constants from a Modified Redlich-Kwong Equation of State[J]. Chem Eng Sci. 1972,27:1197-1203.

[8] LIU Juan-fang, LIU Zhao. The calculations of Moist air's enthalpy and entropy in high temperature and pressure[J]. Jouranal of Engineering Thermophysics, 2007, 28(4):557-560. 